\title{
On the Security of the Winternitz One-Time Signature Scheme Full version ${ }^{\star}$
}

\author{
Johannes Buchmann, Erik Dahmen, Sarah Ereth, Andreas Hülsing*^, and Markus Rückert ${ }^{\star \star \star}$ \\ \{buchmann, dahmen, huelsing, rueckert\}@cdc.informatik.tu-darmstadt.de \\ ereth@mais.informatik.tu-darmstadt.de \\ Technische Universität Darmstadt
}

\begin{abstract}
We show that the Winternitz one-time signature scheme is existentially unforgeable under adaptive chosen message attacks when instantiated with a family of pseudo random functions. Compared to previous results, which require a collision resistant hash function, our result provides significantly smaller signatures at the same security level. We also consider security in the strong sense and show that the Winternitz one-time signature scheme is strongly unforgeable assuming additional properties of the pseudo random function. In this context we formally define several key-based security notions for function families and investigate their relation to pseudorandomness. All our reductions are exact and in the standard model and can directly be used to estimate the output length of the hash function required to meet a certain security level.
\end{abstract}

Keywords Hash-based signatures, post-quantum signatures, pseudorandom functions, security reductions.

\section{Introduction}

Digital signatures are ubiquitous in our computer dominated society. They are basic building blocks of eGovernment and eCommerce. They are used to guarantee the integrity and authenticity of software updates and enable secure Internet connections. The security of currently used signature schemes - RSA and ECDSA - relies on the hardness of certain number theoretic problems, whereas the actual hardness of these problems remains unclear. In 1994 Shor presented a quantum algorithm that can be used to solve the factorization and discrete logarithm problems in polynomial time, thus completely breaking RSA and ECDSA [23]. Given the importance of digital signatures, the search for alternative signature schemes that resist attacks arising from algorithmic and technological advances is an important research goal.

One promising alternative are hash-based signatures. Their sole security requirement is the existence of hash function families with certain properties. Current research suggests, that the security of hash-based signatures will not be significantly harmed by quantum computer supported attacks [12]. Another benefit of hash-based signature schemes is that they are provably secure in the standard model $[6,7,8,13]$. A hash-based signature scheme or Merkle signature scheme (MSS) consists of the combination of a one-time signature scheme (OTS) to sign the data and Merkle's tree authentication scheme [17] which reduces the authenticity of many one-time verification keys to the authenticity of a single public key. Examples for one-time signature schemes are the Lamport-Diffie OTS [14], the Merkle OTS [17], the Winternitz OTS [17,8], the Bleichenbacher-Maurer OTS [3], the

\footnotetext{
* An extended abstract of this paper appears in Proceedings of Africacrypt 2011

${ }^{\star \star}$ Supported by grant no. BU 630/19-1 of the German Research Foundation (www.dfg.de).

$\star \star \star$ Supported by CASED (www. cased.de).
} 
BiBa OTS [18] and HORS [20]. The Winternitz OTS (W-OTS) is most suitable for combining it with Merkle's tree authentication scheme because of the small verification key size and the flexible trade-off between signature size and signature generation time. Further it is possible to compute the corresponding verification key given a W-OTS signature. So a MSS signature does not need to contain the verification key. This is not the case for all of the above mentioned schemes besides the Bleichenbacher-Maurer OTS but it reduces the MSS signature size significantly. Hence efficient variants of the Merkle signature scheme rely on W-OTS [4]. W-OTS is also used for authentication in sensor networks [16].

The size of a Winternitz signature is roughly $m n / w$ bits and signing roughly requires $2^{w} m / w$ hash operations, where $m$ is the bit length of the hash value to be signed, $n$ is the output length of the hash function used in the scheme, and $w$ is the Winternitz parameter determining the tradeoff between signature size and signature generation time. In $[8,13]$, the authors provide security reductions for graph based one-time signature schemes, a general class of OTS which includes WOTS. Due to the generality of graph based OTS, these security reductions require the used hash function to be collision resistant. Collision resistance is one of the strongest security notions of hash functions and admits effective generic attacks using the birthday paradox. Following these reductions, to achieve $b$ bits of security one must use $n=2 b$ and $m=2 b$ which yields W-OTS signatures of size roughly $4 b^{2} / w$ bits.

Our results. In this paper we show that weaker assumptions are sufficient for the security of WOTS. We show that W-OTS is existentially unforgeable under adaptive chosen message attacks [11] when instantiated with a family of pseudorandom functions (PRF). Since the PRF property is not affected by birthday attacks, hash functions with shorter output length can be used which in turn reduces the signature size. This result is especially meaningful because the main issue with hashbased signatures is the signature size. Also, it has been shown that PRF exist if one way functions (OWF) exist $[24,15,10]$ and further, that OWF exist if secure digital signature schemes exist [22]. So our result shows that a secure instance of W-OTS exists, as long as there exists any secure signature scheme. For collision resistant hash function families it is unknown if their existence can be based on the existence of OWF.

We also consider unforgeability in the strong sense by reducing the strong unforgeability of W-OTS to the intractability of finding key collisions (given $x$, find $k, k^{\prime}$ such that $k \neq k^{\prime}$ and $f_{k}(x)=f_{k^{\prime}}(x)$ ) or second keys (given $x$ and key $k$, find $k^{\prime}$ such that $k \neq k^{\prime}$ and $f_{k}(x)=f_{k^{\prime}}(x)$ ). The notion of key collision resistance was used before by the authors of [19] in the security analysis of the TESLA protocol. In [9], the author uses this notion as property of pseudorandom function tribe ensembles to construct a committing and key-hiding private-key encryption scheme. The authors of [5] provide a construction for perfectly one-way functions assuming key collision resistance. We provide a thorough treatment of these key based notions and pseudo randomness. We define them formally and investigate implications and separations among them.

Our results are exact and in the standard model. Such reductions are of enormous practical value compared to asymptotic results or the random oracle model. Exact reductions allow the security level of the scheme to be estimated for fixed security parameters. The standard model uses only security notions which can be efficiently realized in practice. Exact reductions are also of theoretical interest, because they indicate the quality of a reduction and allow an easy comparison of the hardness of the problems. 
Notation. Throughout the paper we stick to the following notation. We use $n$ as the main security parameter. Efficient algorithms require only polynomial time and space in $n$. The statement $x \stackrel{\$}{\longleftarrow} X$ means $x$ is chosen uniformly at random from $X$. The concatenation of strings is done via $\|$. We also write $\log$ for $\log _{2}$. During the paper we measure the runtime of an algorithm in terms of the number of evaluations of the function family used.

Organization. We prove the existential unforgeability of W-OTS using pseudorandom functions in Section 2. We prove the strong unforgeability of W-OTS using second key resistant or key collision resistant functions in Section 3. We examine implications and separations between the introduced security notions in Section 4. We interpret our results and provide concluding remarks in Section 5.

\section{Existential unforgeability of the Winternitz one-time signature scheme}

In this section we prove that the Winternitz one-time signature scheme (W-OTS) is existentially unforgeable under adaptive chosen message attacks (EU-CMA) when instantiated with a family of pseudo-random functions. We begin by reviewing W-OTS and introduce a little tweak required by the reduction. Then we introduce the required security notions. Finally we state the reduction and use it to estimate the security level.

\subsection{The Winternitz one-time signature scheme}

The Winternitz one-time signature scheme was first mentioned in [17] as a generalization of Merkle's OTS also proposed in [17]. A complete description can be found in [8]. The core idea of W-OTS is to iteratively apply a function on a secret input, whereas the number of iterations depends on the message to be signed. The used functions are members of the function family

$$
F(n)=\left\{f_{k}:\{0,1\}^{n} \rightarrow\{0,1\}^{n} \mid k \in\{0,1\}^{n}\right\}
$$

parameterized by key $k \in\{0,1\}^{n}$ and the security parameter $n$. For our purposes iteratively applying a function is defined as follows. We use the output of the function $f_{k}$ as key for the next iteration. The function is always evaluated on the same input $x$. This is in contrast to the original construction, where the output of the function is used as input for the next iteration and the key remains fixed. We use the notation $f_{k}^{i}(x)$ to denote that the function is iterated $i$ times on input $x$ using key $k$ for the first iteration and the output of the function as key for the next iteration, e.g. $f_{k}^{2}(x)=f_{f_{k}(x)}(x)$ and $f_{k}^{0}(x)=x$.

In the following, we only describe the generation of signatures for $m$-bit messages. The generalization to arbitrary sized messages is straight forward by utilizing a collision resistant hash function.

Key pair generation (Algorithm $\mathrm{Kg}$ ). First we choose the Winternitz parameter $w \in \mathbb{N}, w>1$, defining the compression level. Next we choose a random value $x \stackrel{\$}{\longleftarrow}\{0,1\}^{n}$. The signature key consists of $\ell$ bit strings of length $n$ chosen uniformly with the random distribution,

$$
\mathrm{sk}=\left(\mathrm{sk}_{1}, \ldots, \mathrm{sk}_{\ell}\right) \stackrel{\$}{\longleftarrow}\{0,1\}^{(n, \ell)}
$$


where $\ell$ is computed as follows.

$$
\ell_{1}=\left\lceil\frac{m}{\log (w)}\right\rceil, \ell_{2}=\left\lfloor\frac{\log \left(\ell_{1}(w-1)\right)}{\log (w)}\right\rfloor+1, \ell=\ell_{1}+\ell_{2} .
$$

The verification key is computed using functions from the family $F(n)$. The bit strings in the signature key are used as key for the function $f$ and the function is iterated $w-1$ times on input $x$.

$$
\mathrm{pk}=\left(\mathrm{pk}_{0}, \mathrm{pk}_{1}, \ldots, \mathrm{pk}_{\ell}\right)=\left(x, f_{\mathrm{sk}_{1}}^{w-1}(x), \ldots, f_{\mathrm{sk}_{\ell}}^{w-1}(x)\right)
$$

Signature generation (Algorithm Sign.) We describe how to sign an $m$-bit message $M=\left(M_{1}, \ldots, M_{\ell_{1}}\right)$ given in base- $w$ representation, i.e. $M_{i} \in\{0, \ldots, w-1\}$ for $i=1, \ldots, \ell_{1}$. We begin by computing the checksum

$$
C=\sum_{i=1}^{\ell_{1}}\left(w-1-M_{i}\right)
$$

and represent it to base $w$ as $C=\left(C_{1}, \ldots, C_{\ell_{2}}\right)$. The length of the base- $w$ representation of $C$ is at most $\ell_{2}$ since $C \leq \ell_{1}(w-1)$. Then we set $B=\left(b_{1}, \ldots, b_{\ell}\right)=M \| C$. The signature of message $M$ is computed as

$$
\sigma=\left(\sigma_{1}, \ldots, \sigma_{\ell}\right)=\left(f_{\mathbf{s k}_{1}}^{b_{1}}(x), \ldots, f_{\text {sk }_{\ell}}^{b_{\ell}}(x)\right) .
$$

Signature verification (Algorithm $\vee f$.) The verifier first computes the base- $w$ string $B=\left(b_{1}, \ldots, b_{\ell}\right)$ as described above. Then he checks whether

$$
\left(f_{\sigma_{1}}^{w-1-b_{1}}\left(\mathrm{pk}_{0}\right), \ldots, f_{\sigma_{\ell}}^{w-1-b_{\ell}}\left(\mathrm{pk}_{0}\right)\right) \stackrel{?}{=}\left(\mathrm{pk}_{1}, \ldots, \mathrm{pk}_{\ell}\right) .
$$

The signature is accepted iff the comparison holds.

\subsection{Security notions for signature schemes and function families}

We begin by reviewing the standard definition of digital signature schemes and the security notion existential unforgeability under adaptive chosen message attacks (EU-CMA) [11]. We then define two security notions for function families required for our reduction. The first is the well known pseudo-randomness property. The second is key one-wayness which states that it is hard to find a key $k$ such that the function $f_{k}$ maps a given input $x$ to a given output $y$. We also state two lemmas about these notions which will be useful for the reduction of W-OTS.

Definition 1 (Digital signature schemes). A digital signature scheme $\mathrm{Sig}=(\mathrm{Kg}, \mathrm{Sign}, \mathrm{Vf})$ is a triple of PPT algorithms:

- $\operatorname{Kg}\left(1^{n}\right)$ on input of a security parameter $1^{n}$ outputs a private signing key sk and a public verification key $\mathrm{pk}$;

- Sign(sk, $M)$ outputs a signature $\sigma$ under sk for the message $M$;

- $\mathrm{Vf}(\mathrm{pk}, \sigma, M)$ outputs 1 iff $\sigma$ is a valid signature on $M$ under $\mathrm{pk}$.

Definition 2 (Existential unforgeability (EU-CMA)). EU-CMA is defined by the following experiment. 


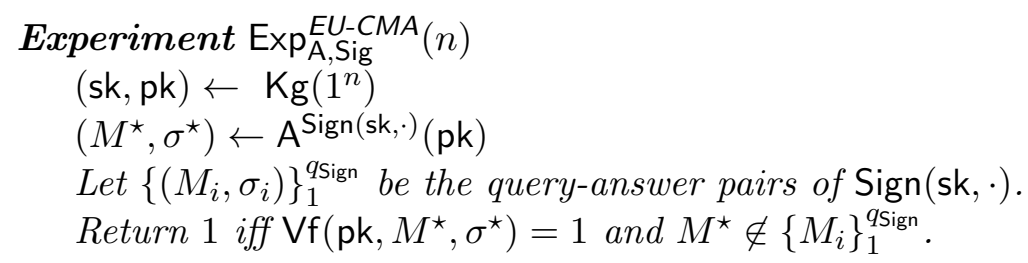

Sig is $(t, \epsilon, q)$-existentially unforgeable if there is no t-time adversary that succeeds with probability $\geq \epsilon$ after making $\leq q$ signature oracle queries.

A $(t, \epsilon, 1)$-EU-CMA secure signature scheme is called one-time signature scheme.

Definition 3 (Pseudorandom functions (PRF)). A family of functions $F(n)$ is pseudorandom, if no efficient algorithm Dis is able to distinguish a randomly chosen function $f_{k} \in F(n)$ from a randomly chosen function from the set $G(n)$ of all functions with same domain and range as $F(n)$. The formal definition is taken from [2]. Dis gets access to an oracle Box $(\cdot)$ implementing a function randomly chosen from $F(n)$ or $G(n)$ in a black box manner. The distinguisher may adaptively query $\operatorname{Box}(\cdot)$ as often as he likes. Finally, the distinguisher outputs 1 if he thinks that Box models a function from $F(n)$ and 0 otherwise.

Let $F(n)$ be a family of functions as in (1) and $G(n)=\left\{g:\{0,1\}^{n} \rightarrow\{0,1\}^{n}\right\}$ the family of all functions with domain and range $\{0,1\}^{n}$. We call $F(n)(t, \epsilon)-\mathrm{PRF}$, if the advantage

$$
\operatorname{Adv}_{F(n)}^{\mathrm{PRF}}(\operatorname{Dis})=\left|\operatorname{Pr}\left[\operatorname{Box} \stackrel{\$}{\longleftarrow} F(n): \operatorname{Dis}^{\operatorname{Box}(\cdot)}=1\right]-\operatorname{Pr}\left[\operatorname{Box} \stackrel{\$}{\longleftarrow} G(n): \operatorname{Dis}^{\operatorname{Box}(\cdot)}=1\right]\right|
$$

of any distinguisher Dis that runs in time $t$ is at most $\epsilon$.

Definition 4 (Key one-wayness (KOW)). Let $F(n)$ be a family of functions as in (1). We call $F(n)(t, \epsilon)-\mathrm{KOW}$, if the success probability

$$
\operatorname{Adv}_{\mathrm{A}}^{\mathrm{KOW}}=\operatorname{Pr}\left[(x, k) \stackrel{\$}{\longleftarrow}\{0,1\}^{n} \times\{0,1\}^{n}, y \leftarrow f_{k}(x), k^{\prime} \longleftarrow \mathrm{A}(x, y): y=f_{k^{\prime}}(x)\right]
$$

of any adversary $\mathrm{A}$ that runs in time $t$ is at most $\epsilon$.

Please recall, that the time $t$ is counted in terms of evaluations of $f$. We assume, that a call to Box takes the same time as an evaluation of $f$. The security level or bit security $b$ the family $F(n)$ or a signature scheme Sig provides against attacks on the respective notion is computed as $b=\log (t / \epsilon)$.

A key collision of $F(n)$ is defined as a pair of distinct keys $\left(k, k^{\prime}\right)$ such that $f_{k}(x)=f_{k^{\prime}}(x)$ holds for some $x \in\{0,1\}^{n}$. We define an upper $(\kappa)$ and lower $\left(\kappa^{\prime}\right)$ bound on the number of key collisions that occur in the family $F(n)$.

Definition 5. The upper bound $\kappa$ is defined as follows: For each pair $(x, k)$, there exist at most $\kappa-1$ different keys $k_{1}, \ldots, k_{\kappa-1}$, which are also different from $k$, such that $f_{k}(x)=f_{k_{i}}(x)$ for $i=1, \ldots, \kappa-1$. Equivalently we define the lower bound $\kappa^{\prime}:$ For each pair $(x, k)$, there exist at least $\kappa^{\prime}-1$ different keys $k_{1}, \ldots, k_{\kappa^{\prime}-1}$, which are also different from $k$, such that $f_{k}(x)=f_{k_{i}}(x)$ for $i=1, \ldots, \kappa^{\prime}-1$. 
The values $\kappa$ and $\kappa^{\prime}$ restrict the number of different images $y$ some preimage $x$ can be mapped to by functions in $F(n)$, i.e.

$$
\frac{2^{n}}{\kappa} \leq\left|\left\{f_{k}(x): k \in\{0,1\}^{n}\right\}\right| \leq \frac{2^{n}}{\kappa^{\prime}}
$$

for all $x \in\{0,1\}^{n}$. Also, given $y \stackrel{\$}{\longleftarrow}\{0,1\}^{n}$ the probability that there exists a key $k$ and preimage $x$ such that $f_{k}(x)=y$ holds is at least $1 / \kappa$.

The following lemma describes an interesting relation between the security level of pseudorandom functions and the value $\kappa$ defined above.

Lemma 1. Let $F(n)$ be $(t, \epsilon)-\mathrm{PRF}$ with security level $b=\log (t / \epsilon)$ and $\kappa$ as in Definition 5. Then $\kappa \leq 2^{n-b}+1$.

Proof. Assume $\kappa>2^{n-b}+1$ and let $(x, y)$ be a pair where there exist $\kappa$ keys mapping $x$ to $y$. The distinguisher Dis queries Box with $x$. If $\operatorname{Box}(x)=y$ then Dis returns 1 and 0 otherwise. Clearly Dis runs in time $t^{\prime}=1$. Further we have $\operatorname{Pr}\left[\operatorname{Box} \stackrel{\$}{\longleftarrow} F(n): \operatorname{Dis}^{\operatorname{Box}(\cdot)}=1\right]=\kappa / 2^{n}>2^{-b}+2^{-n}$ and $\operatorname{Pr}\left[\operatorname{Box} \stackrel{\$}{\longleftarrow} G: \operatorname{Dis}^{\operatorname{Box}(\cdot)}=1\right]=2^{-n}$ and therefore $\epsilon^{\prime}=\operatorname{Adv}_{F(n)}^{\mathrm{PRF}}($ Dis $)>2^{-b}$ which is a contradiction.

The following lemma states that the KOW property is implied by the PRF property. In other words, an efficient attacker against the KOW property leads to an efficient distinguisher.

Proposition 1 (PRF $\Rightarrow$ KOW). Let $F(n)$ be $(t, \epsilon)$-PRF. Then $F(n)$ is $\left(t-2, \epsilon /\left(1 / \kappa-1 / 2^{n}\right)\right.$ KOW.

Proof. Assume there exists an adversary $\mathrm{A}_{\mathrm{KOW}}(x, y)$ who finds a key $k$ satisfying $y=f_{k}(x)$ in time $t_{\text {KOW }}$ with probability $\epsilon_{\mathrm{KOW}}$. Then we can construct a distinguisher Dis using $\mathrm{A}_{\mathrm{KOW}}$ the following way: Dis queries $\operatorname{Box}(\cdot)$ with $x \in\{0,1\}^{n}$. After receiving the answer $y$, Dis runs $\mathrm{A}_{\mathrm{KOW}}(x, y)$ to obtain key $k$. Then Dis queries Box with a second value $x^{\prime} \in\{0,1\}^{n}$. If $\operatorname{Box}\left(x^{\prime}\right)=f_{k}\left(x^{\prime}\right)=y^{\prime}$ Dis returns 1 and 0 otherwise. In case Box $\stackrel{\$}{\longleftarrow} F(n)$, the probability that $\mathrm{A}_{\text {KOW outputs a key } k \text { such }}$ that $f_{k}(x)=y$ holds is $\epsilon_{\mathrm{KOW}}$. The probability that $f_{k}\left(x^{\prime}\right)=y^{\prime}$ holds is at least $1 / \kappa$, because at least one of the $\kappa$ functions in $F(n)$ mapping $x$ to $y$ also maps $x^{\prime}$ to $y^{\prime}$. In case Box $\stackrel{\$}{\longleftarrow} G(n)$, the probability that $\mathrm{A}_{\mathrm{KOW}}$ outputs a key $k$ such that $f_{k}(x)=y$ holds is at most $\epsilon_{\mathrm{KOW}}$. The probability that $f_{k}\left(x^{\prime}\right)=y^{\prime}$ holds is $1 / 2^{n}$, because from the $2^{n\left(2^{n}-1\right)}$ functions in $G$ mapping $x$ to $y$, only $2^{n\left(2^{n}-2\right)}$ also map $x^{\prime}$ to $y^{\prime}$. In summary we get $\epsilon \geq \operatorname{Adv}_{F(n)}^{\mathrm{PRF}}($ Dis $) \geq \epsilon_{\mathrm{KOW}}\left(1 / \kappa-1 / 2^{n}\right)$.

\subsection{Security reduction}

We now state the main result of this section.

Theorem 1. Let $F(n)$ be a family of functions as in Equation (1) and $\kappa$ as in Definition 5. If $F(n)$ is $\left(t_{\mathrm{PRF}}, \epsilon_{\mathrm{PRF}}\right)-\mathrm{PRF}$ then $W$-OTS is $(t, \epsilon, 1)$ EU-CMA with

$$
\begin{aligned}
& t=t_{\mathrm{PRF}}-t_{\mathrm{Kg}}-t_{\mathrm{Vf}}-2 \\
& \epsilon \leq \epsilon_{\mathrm{PRF}} \ell^{2} w^{2} \kappa^{w-1} \frac{1}{\left(\frac{1}{\kappa}-\frac{1}{2^{n}}\right)}
\end{aligned}
$$


Proof. The proof works as follows: First we use a forger for W-OTS to construct an adversary on the key one wayness of $F(n)$. This adversary is then used to construct a distinguisher using Proposition 1. Algorithm 1 shows how a forger For ${ }^{\operatorname{Sign}(\mathrm{sk}, \cdot)}$ (pk) for W-OTS can be used to construct an adversary $\mathrm{A}_{\mathrm{KOW}}$ on the key one-wayness of $F(n)$. The signing oracle Sign is simulated by the adversary.

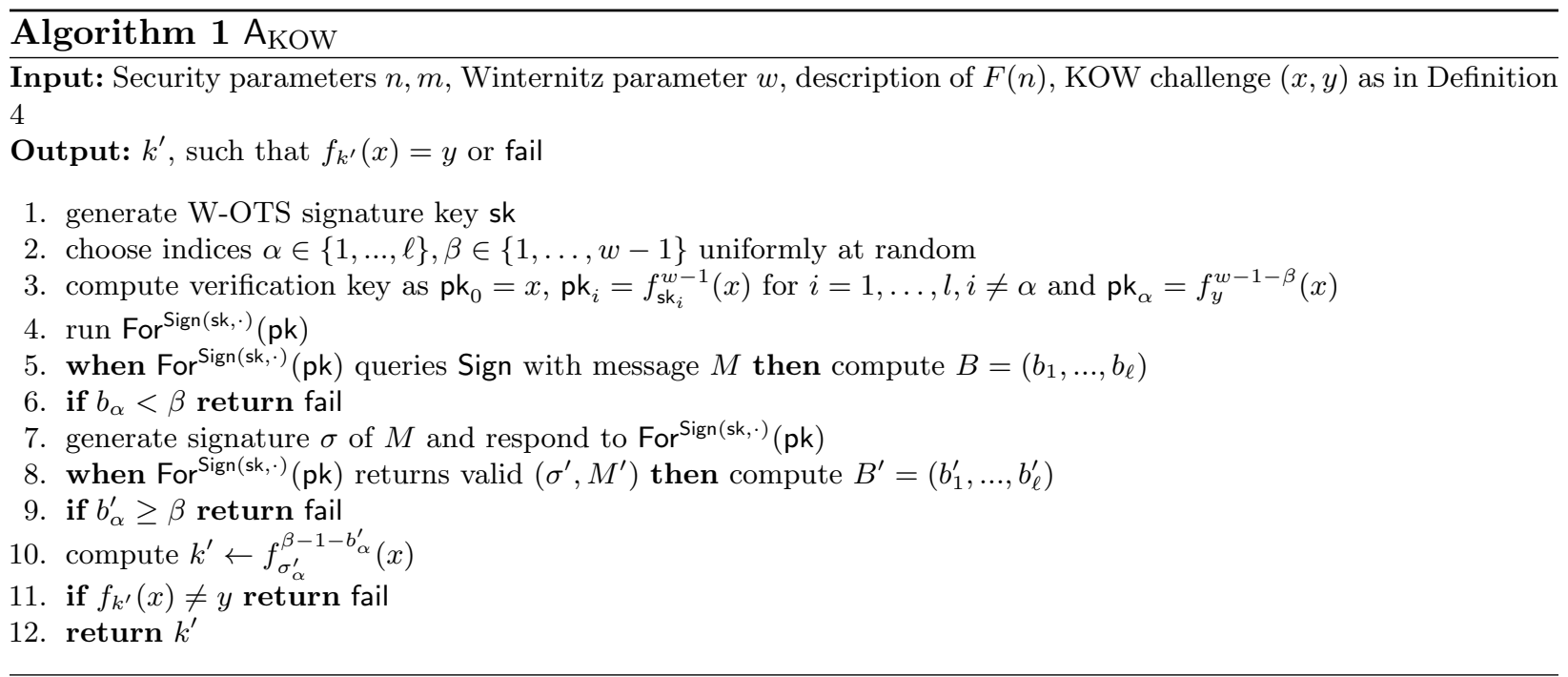

The goal of the adversary $\mathrm{A}_{\mathrm{KOW}}$ is to produce a key $k^{\prime}$ such that $f_{k^{\prime}}(x)=y$ for $x, y$ provided as input. $A_{\text {KOW }}$ begins by generating a regular W-OTS signature key pair and choosing random positions $\alpha$ and $\beta$ (Lines 1,2). Then he computes the W-OTS verification key using value $x$. The bit string at position $\alpha$ in the verification key $\left(\mathrm{pk}_{\alpha}\right)$ is computed by inserting $y$ at position $\beta$ in the hash chain used to compute $\mathrm{pk}_{\alpha}$ (Line 3). Next, $\mathrm{A}_{\mathrm{KOW}}$ calls the forger and waits for it to ask an oracle query. The forgers query can only be answered if $b_{\alpha} \geq \beta$ holds, because $A_{\mathrm{KOW}}$ doesn't know the first $\beta$ entries in the corresponding hash chain (Line 6 ). The forgery produced by the forger is only meaningful to $\mathrm{A}_{\mathrm{KOW}}$ if $b_{\alpha}^{\prime}<\beta$ holds (Line 9). Only then the bit string $\sigma_{\alpha}$ in the forged signature might yield a key $k^{\prime}$ such that $y=f_{k^{\prime}}(x)$ holds (Lines 10,11). We now compute the success probability of $A_{\text {KOW }}$. W.l.o.g we assume that the forger queries the signing oracle. The probability of $b_{\alpha} \geq \beta$ in Line 6 is at least $(\ell w)^{-1}$. This is because of the checksum which guarantees that not all of the $b_{i}$ are zero simultaneously. The probability that the forger succeeds in Line 8 is at least $\epsilon$ by definition. This probability holds under the condition that the verification key pk computed in Line 3 resembles a regular verification key which is the case if there exists a key $k$ such that $f_{k}^{\beta}(x)=y$. This happens with probability at least $1 / \kappa^{\beta}$ according to Definition 5 . The probability of $b_{\alpha}^{\prime}<\beta$ in Line 9 is at least $(\ell w)^{-1}$. This is because of $M \neq M^{\prime}$ and the checksum which guarantees that $b_{i}>b_{i}^{\prime}$ for some $i \in\{1, \ldots, \ell\}$. The probability that $y=f_{k^{\prime}}(x)$ holds in Line 11 is at least $1 / \kappa^{w-1-\beta}$. This is because there exist $\kappa^{w-1}$ keys mapping $x$ to pk ${ }_{\alpha}$ after $w-1$ iterations and only $\kappa^{\beta}$ of these keys map $x$ to $y$ after $\beta$ iterations.

In summary we have $\epsilon_{\mathrm{KOW}} \geq \epsilon /\left(\ell^{2} w^{2} \kappa^{\beta} \kappa^{w-1-\beta}\right)$ and $t_{\mathrm{KOW}}=t+t_{\mathrm{Kg}}+t_{\mathrm{Vf}}$ as the time for the signature query is already taken into account at the runtime of the forger. Combining this with 
Proposition 1 yields $\epsilon_{\mathrm{PRF}} \geq \epsilon\left(1 / \kappa-1 / 2^{n}\right) /\left(\ell^{2} w^{2} \kappa^{w-1}\right)$ and $t_{\mathrm{PRF}}=t+t_{\mathrm{Kg}}+t_{\mathrm{Vf}}+2$ which concludes the proof.

\subsection{Security level}

We now compute the security level of W-OTS for the case that only generic attacks against the PRF property of the function family $F(n)$ exist.

Corollary 1. Let $b=\log (t / \epsilon)$ denote the security level and use $\ell w$ as upper bound for $t_{\mathrm{Kg}}$ and $t_{\mathrm{Vf}}$, respectively. Let $F(n)$ be $\left(2^{n-1-\log \kappa}, 1 / 2\left(1 / \kappa-1 / 2^{n}\right)\right)-\mathrm{PRF}$ with $\kappa=2$. Then the security level of $W$-OTS is

$$
b \geq n-w-1-2 \log (\ell w)
$$

Proof. We use a $\left(t_{\mathrm{PRF}}, \epsilon_{\mathrm{PRF}}\right)$-PRF family $F(n)$ and assume that the best attack on the pseudorandomness of $F(n)$ is a brute-force key recovery attack. An attacker that searches through $t_{\mathrm{KOW}}=2^{n-1-\log \kappa}$ keys has success probability $\epsilon_{\mathrm{KOW}}=1 / 2$ for recovering the correct key. By Proposition 1 this yields an $t_{\mathrm{PRF}}=2^{n-1-\log \kappa}+2, \epsilon_{\mathrm{PRF}}=1 / 2\left(1 / \kappa-1 / 2^{n}\right)$ distinguisher for the pseudorandomness of $F(n)$. The security level of the PRF property of $F(n)$ in presence of this distinguisher is $b=n$ which in turn implies $\kappa \leq 2$ according to Lemma 1. The security level of W-OTS using $F(n)$ is computed as follows

$$
\begin{aligned}
2^{b}=\frac{t}{\epsilon} & \geq \frac{t_{\mathrm{PRF}}-t_{\mathrm{Kg}}-t_{\mathrm{Vf}}-2}{\epsilon_{\mathrm{PRF}} \ell^{2} w^{2} \kappa^{w-1}}\left(\frac{1}{\kappa}-\frac{1}{2^{n}}\right) \\
& \geq \frac{2^{n-\log \kappa}-4 \ell w}{\ell^{2} w^{2} \kappa^{w-1}} \\
& \geq 2^{n-w-2 \log (\ell w)}-\frac{4}{\ell w 2^{w-1}}
\end{aligned}
$$

Since $4 /\left(\ell w 2^{w-2}\right) \leq 2^{n-w-1-2 \log (\ell w)}$ for all reasonable choices of $w$ and $m$ we finally obtain $b \geq$ $n-w-1-2 \log (\ell w)$ as security level of W-OTS.

\section{Strong unforgeability of the Winternitz one-time signature scheme}

While the reduction of the last section shows that W-OTS is EU-CMA assuming a standard security notion for hash functions, it does not provide security in the strong sense. This is accomplished by two reductions presented in this section. We show that W-OTS is strongly unforgeable under adaptive chosen message attacks (SU-CMA), if the used function family is either second key resistant or key collision resistant. The difference between EU-CMA and SU-CMA is, that in SU-CMA the adversary also wins if he returns a new signature for an already queried message. While these reductions provide stronger security guarantees, they do not rely on standard security notions of hash functions. One is therefore confronted with a trade-off between security and requirements on the hash function. Again we begin by introducing the required security notions and then continue with the reductions and the computation of the security levels. 


\subsection{Security notions for signature schemes and function families II}

We begin by reviewing the definition of strong unforgeability under adaptive chosen message attacks. Then, we define two security notions for function families required for our reductions. The first is second key resistance which states that given key $k$ and preimage $x$, it is hard to find a key $k^{\prime} \neq k$ such that $f_{k}(x)=f_{k^{\prime}}(x)$. The second is key collision resistance which states that given preimage $x$, it is hard to find two distinct keys $k, k^{\prime}$ such that $f_{k}(x)=f_{k^{\prime}}(x)$.

Definition 6 (Strong unforgeability (SU-CMA)). SU-CMA is defined by the following experiment.

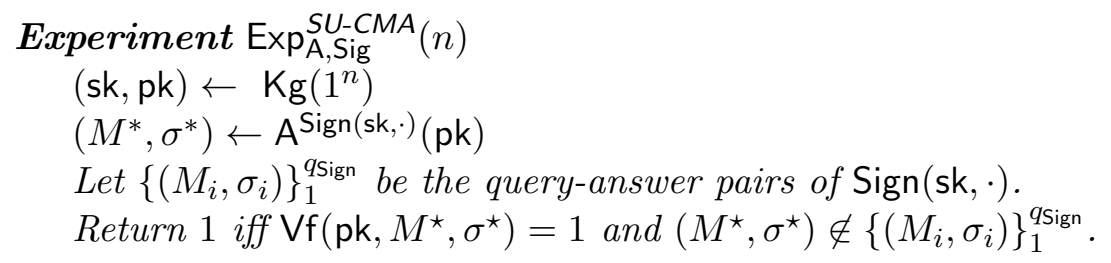

The signature scheme $\operatorname{Sig}$ is $(t, \epsilon, q)$-SU-CMA if there is no t-time adversary that succeeds with probability $\geq \epsilon$ after making $\leq q$ signature oracle queries.

Definition 7 (Second key resistance (SKR)). Let $F(n)$ be a family of functions as in (1). We call $F(n)(t, \epsilon)$-SKR, if the success probability

$$
\mathrm{Adv}_{\mathrm{A}}^{\mathrm{SKR}}=\operatorname{Pr}\left[(x, k) \stackrel{\$}{\longleftarrow}\{0,1\}^{n} \times\{0,1\}^{n}, k^{\prime} \leftarrow \mathrm{A}(x, k): k^{\prime} \neq k, f_{k^{\prime}}(x)=f_{k}(x)\right]
$$

of any adversary $\mathrm{A}$ that runs in time $t$ is at most $\epsilon$.

Definition 8 (Key collision resistance (KCR)). Let $F(n)$ be a a family of functions as in (1). We call $F(n)(t, \epsilon)$-KCR, if the success probability

$$
\mathrm{Adv}_{\mathrm{A}}^{\mathrm{KCR}}=\operatorname{Pr}\left[x \stackrel{\$}{\longleftarrow}\{0,1\}^{n},\left(k, k^{\prime}\right) \leftarrow \mathrm{A}(x): k \neq k^{\prime}, f_{k}(x)=f_{k^{\prime}}(x)\right]
$$

of any adversary $\mathrm{A}$ that runs in time $t$ is at most $\epsilon$.

Proposition $2(\mathrm{SKR} \Rightarrow \mathrm{KOW})$. Let $F(n)$ be $(t, \epsilon)$-SKR with $\kappa^{\prime}>1$. Then $F(n)$ is $(t-1, \epsilon /(1-$ $\left.\left.1 / \kappa^{\prime}\right)\right)$-KOW .

Proof. Towards contradiction, let us assume a successful adversary A that breaks KOW for $F(n)$. We show how to use $\mathrm{A}$ as a black-box in an algorithm $\mathcal{B}$ to break $\mathrm{SKR}$. On input $(x, k)$ from the SKR experiment, the algorithm $\mathcal{B}$ computes $y \leftarrow f_{k}(x)$ and runs $\mathrm{A}(x, y)$. The subroutine returns $k^{\prime}$ such that $f_{k}(x)=f_{k^{\prime}}(x)$ with probability at least $\epsilon$. Then, $\mathcal{B}$ returns $k^{\prime}$. Since $\kappa^{\prime}(F(n))>1$, the algorithm $A$ returns a key that is different from $k$ with probability at least $1-1 / \kappa^{\prime} \geq 1 / 2$. Thus, $\mathcal{B}$ is successful with probability $\epsilon\left(1-1 / \kappa^{\prime}\right) . \kappa^{\prime}>1$ is required to guarantee that a different key actually exists. 


\subsection{Security reductions}

We now state the main result of this section.

Theorem 2. Let $F(n)$ be a family of functions as in Equation (1) and $\kappa, \kappa^{\prime}$ as in Definition 5. a) If $F(n)$ is $\left(t_{\mathrm{SKR}}, \epsilon_{\mathrm{SKR}}\right)$-SKR then $W$-OTS is $(t, \epsilon, 1) S U$-CMA with

$$
\begin{aligned}
& t \geq t_{\mathrm{SKR}}-t_{\mathrm{Kg}}-t_{\mathrm{Vf}}-1 \\
& \epsilon \leq \epsilon_{\mathrm{SKR}} \ell^{2} w^{2} \kappa^{w-2} \frac{\kappa^{\prime}}{\kappa^{\prime}-1}
\end{aligned}
$$

b) If $F(n)$ is $\left(t_{\mathrm{KCR}}, \epsilon_{\mathrm{KCR}}\right)$-KCR then $W$-OTS is $(t, \epsilon, 1) S U$-CMA with

$$
\begin{aligned}
& t \geq t_{\mathrm{KCR}}-t_{\mathrm{Kg}}-t_{\mathrm{Vf}} \\
& \epsilon \leq \epsilon_{\mathrm{KCR}} \frac{\kappa^{\prime}}{\kappa^{\prime}-1}
\end{aligned}
$$

The proof of this theorem can be found in appendix A

\subsection{Security level}

We now compute the security level of W-OTS for the case that only generic attacks against the SKR or KCR property of the function family $F(n)$ exist.

Note, that in case of $\kappa=1$ it is impossible to find two signatures for the same message by construction. Therefore W-OTS is SU-CMA secure if it is EU-CMA secure and $\kappa=1$. For the computation of the security level in this section we therefore assume $\kappa, \kappa^{\prime} \geq 2$, such that there exists at least one key collision for each preimage.

Corollary 2. Let $b=\log (t / \epsilon)$ denote the security level and use $\ell w$ as upper bound for $t_{\mathrm{Kg}}$ and $t_{\mathrm{Vf}}$, respectively.

a) Let $F(n)$ be $\left(2^{n-1-\log \kappa}+1,\left(\kappa^{\prime}-1\right) /\left(2 \kappa^{\prime}\right)\right)-\mathrm{SKR}$ and $\left(t_{\mathrm{PRF}}, \epsilon_{\mathrm{PRF}}\right)-\mathrm{PRF}$ with $\log \left(t_{\mathrm{PRF}} / \epsilon_{\mathrm{PRF}}\right)=n$ and $\kappa^{\prime}=\kappa=2$. Then the security level of $W$-OTS is

$$
b \geq n-w-2 \log (\ell w)
$$

b) Let $F(n)$ be $\left(2^{\left(n-\log \kappa^{\prime}\right) / 2}, 1 / 2\right)-\mathrm{KCR}$ and $\left(t_{\mathrm{PRF}}, \epsilon_{\mathrm{PRF}}\right)-\mathrm{PRF}$ with $\log \left(t_{\mathrm{PRF}} / \epsilon_{\mathrm{PRF}}\right)=n$ and $\kappa^{\prime}=$ $\kappa=2$. Then the security level of $W$-OTS is

$$
b \geq(n-1) / 2-1
$$

Proof. a) We use a $\left(t_{\mathrm{SKR}}, \epsilon_{\mathrm{SKR}}\right)$-SKR family $F(n)$ and assume that the best attack on the second key resistance of $F(n)$ is a brute-force key recovery attack. An attacker that searches through $t_{\mathrm{KOW}}=2^{n-1-\log \kappa}$ keys has success probability $\epsilon_{\mathrm{KOW}}=1 / 2$ for recovering the correct key. By Proposition 2 this yields an

$$
t_{\mathrm{SKR}}=2^{n-1-\log \kappa}+1, \epsilon_{\mathrm{SKR}}=\frac{1}{2} \cdot \frac{\kappa^{\prime}-1}{\kappa^{\prime}}
$$


adversary on the second key resistance of $F(n)$. The security level of the SKR property of $F(n)$ in presence of this adversary is $b=n-\log (\kappa-1)$, assuming $\kappa=\kappa^{\prime}$. We further assume that $F(n)$ is $\left(t_{\mathrm{PRF}}, \epsilon_{\mathrm{PRF}}\right)$-PRF with $\log \left(t_{\mathrm{PRF}} / \epsilon_{\mathrm{PRF}}\right)=n$. This justifies using $\kappa^{\prime}=\kappa=2$ since $\kappa^{\prime} \geq 2$ is required to ensure that second keys actually exist. The security level of W-OTS is computed as follows

$$
\begin{aligned}
2^{b}=\frac{t}{\epsilon} & \geq \frac{t_{\mathrm{SKR}}-t_{\mathrm{Kg}}-t_{\mathrm{Vf}}-1}{\epsilon_{\mathrm{SKR}} \ell^{2} w^{2} \kappa^{w-2}} \cdot \frac{\kappa^{\prime}-1}{\kappa^{\prime}} \\
& =\frac{2^{n-\log \kappa}-4 \ell w}{\ell^{2} w^{2} \kappa^{w-2}} \cdot \frac{\kappa^{\prime}-1}{\kappa^{\prime}} \cdot \frac{\kappa}{\kappa-1} \\
& \geq 2^{n-w+1-2 \log (\ell w)}-\frac{4}{\ell w 2^{w-2}}
\end{aligned}
$$

Since $4 /\left(\ell w 2^{w-2}\right) \leq 2^{n-w-2 \log (\ell w)}$ for all reasonable choices of $w$ and $m$ we finally obtain $b \geq$ $n-w-2 \log (\ell w)$ as security level of W-OTS.

b) We use a $\left(t_{\mathrm{KCR}}, \epsilon_{\mathrm{KCR}}\right)$-KCR family $F(n)$ and assume that the best attack on the key collision resistance of $F(n)$ is a birthday attack, i.e. an adversary that searches through $t_{\mathrm{KCR}}=2^{\left(n-\log \kappa^{\prime}\right) / 2}$ keys has success probability $\epsilon_{\mathrm{KCR}}=1 / 2$ for finding a key collision. The security level of the KCR property of $F(n)$ in presence of this adversary is $b=\left(n-\log \kappa^{\prime}\right) / 2-1$. Again we assume that $F(n)$ is $\left(t_{\mathrm{PRF}}, \epsilon_{\mathrm{PRF}}\right)-\mathrm{PRF}$ with $\log \left(t_{\mathrm{SKR}} / \epsilon_{\mathrm{SKR}}\right)=n$ and use $\kappa^{\prime}=\kappa=2$. The security level of W-OTS is computed as follows

$$
2^{b}=\frac{t}{\epsilon} \geq \frac{t_{\mathrm{KCR}}-t_{\mathrm{Kg}}-t_{\mathrm{Vf}}}{\epsilon_{\mathrm{KCR}}} \cdot \frac{\kappa^{\prime}-1}{\kappa^{\prime}} \geq 2^{(n-1) / 2}-2 \ell w
$$

Since $2 \ell w \leq 2^{(n-1) / 2-1}$ for reasonable choices of $w$ and $m$ we finally obtain $b \geq(n-1) / 2-1$ as security level of W-OTS.

\section{Relation between security notions}

In this section we complete the analysis of implications and separations between key one-wayness (KOW), second key resistance (SKR), key collision resistance (KCR), and pseudorandomness (PRF) started with Propositions 1 and 2, whereas the suspected separation PRF $\nRightarrow$ SKR is left as an open problem. The proofs of this section can be found in Appendix B. Figure 1 summarizes our findings.

Proposition 3 (KOW $\nRightarrow \mathrm{PRF}$ ). Let $g:\{0,1\}^{n} \rightarrow\{0,1\}^{n}$ be a one-way function. Then there exists a family $F(n)$ that is KOW but not PRF.

Proposition 4 (KOW $\nRightarrow \mathrm{SKR})$. Let $F(n)$ be $(t, \epsilon)$-KOW. Then, there is a family $F^{\prime}(n)$ that is $(t, 2 \epsilon)$-KOW but not SKR.

Proposition 5 (KOW $\nRightarrow \mathrm{KCR}$ ). Let $F(n)$ be $(t, \epsilon)$-KOW. Then, there is a family $F^{\prime}(n)$ of functions that is $\left(t, \epsilon+2 / 2^{n}\right)$-KOW but not KCR.

Proposition $6(\mathrm{KCR} \Rightarrow \mathrm{SKR})$. Let $F(n)$ be $(t, \epsilon)$-KCR. Then $F(n)$ is $(t, \epsilon)$-SKR.

Proposition 7 (SKR $\nRightarrow \mathrm{KCR).} \mathrm{Let} F(n)$ be $(t, \epsilon)$-SKR. Then, there is a family $F^{\prime}(n)$ of functions that is $\left(t, \epsilon+2 / 2^{n}\right)$-SKR but not KCR. 


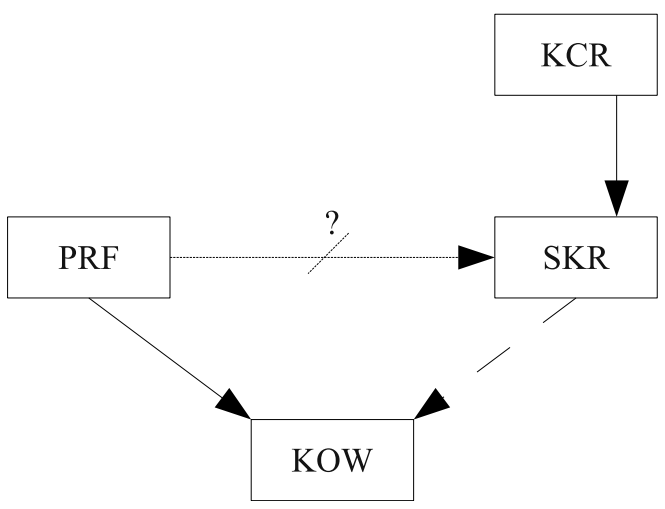

Fig. 1. Implications among PRF, KOW, SKR, and KCR. A straight arrow $A \rightarrow B$ means that property $A$ implies property $B$ and a dashed line means that the implication is conditional. When there is no arrow, it means that we show a separation. The suspected separation between PRF and SKR is an open problem.

Proposition $8(\mathrm{PRF} \nRightarrow \mathrm{KCR})$. Let $F(n)$ be $(t, \epsilon)-\mathrm{PRF}$. Then, there is a family $F^{\prime}(n)$ of functions that is $\left(t, \epsilon+2 / 2^{n}\right)-\mathrm{PRF}$ but not KCR.

The following corollaries can be proven in analogy to Proposition 3.

Corollary 3 (SKR $\nRightarrow \mathrm{PRF).} \mathrm{If} \mathrm{second} \mathrm{preimage} \mathrm{resistant} \mathrm{functions} \mathrm{exist,} \mathrm{there} \mathrm{is} \mathrm{a} \mathrm{family} F(n)$ that is SKR but not PRF.

Corollary 4 (KCR $\nRightarrow \mathrm{PRF})$. If collision resistant functions exist, there is a family $F(n)$ that is KCR but not PRF.

\section{Conclusion}

We have provided three security reductions for W-OTS. The first one shows that W-OTS provides a security level of at least $n-w-1-2 \log (\ell w)$, if the security level of the PRF property of the used function family is at least $n$. When using $n=128$ and $w=16$ the security level of W-OTS is at least 91 while the size of a signature is 560 Bytes. The more conservative approach of using $n=160$ yields a security level of at least 129, which guarantees long-term security but results in larger signatures of 860 Bytes. This reduction is especially appealing because it only assumes a standard security notion of hash functions. SHA-1 and SHA-2 being PRFs is required when using them in the HMAC construction and SHA-3 will be specifically designed to be a PRF. Furthermore, this reduction also works for the special class of pseudorandom permutations (PRP). PRP is the standard model for block ciphers, so it is possible to replace the hash function family with a block cipher. As a block cipher with $n$ bit keys is normally assumed to provide $n$ bit security against distinguishing attacks this justifies our assumption of $\kappa \leq 2$ given Lemma 1. Since several of today's CPUs are equipped with an AES co-processor, this might also lead significant speed-ups in practice.

However, this reduction does not guarantee strong unforgeability, except in case of $\kappa=1$ meaning that no key collisions exist. If no key collisions exist, each message has a unique signature and the scheme is trivially SU-CMA when it is EU-CMA. Showing SU-CMA in general requires that the underlying functions are either SKR or KCR. This has been shown in the second and third 
reduction. The security level of $\mathrm{W}$-OTS is at least $n-w-1-2 \log (\ell w)$ if the security level of the SKR property of the used PRF is at least $n-\log (\kappa-1)$. When using KCR, the security level of W-OTS is at least $(n-1) / 2-1$ if the security level of the KCR property of the used PRF is at least $(n-\log \kappa) / 2-1$. We remark that the last reduction also works with the original Winternitz construction using a family of collision resistant hash functions. In other words, W-OTS is SU-CMA if the used function is collision resistant. However, using a PRF with additional KCR property has the benefit that an exact value for the maximum number $\kappa$ of key collisions that occur within the family is known. This is required for the estimation of the exact security level.

As a by-product we have defined three key-based security notions for function families: key one-wayness (KOW), second key resistance (SKR), and key collision resistance (KCR). We have analyzed implications and separations among these properties and pseudorandomness. Although, these relations have not been analyzed before, they support the common intuition. In fact, keybased and non-key-based notions share an analoguous hierarchy of implications and separations with respect to preimage resistance, second preimage resistance, and collision resistance. We refer the reader to [21] for a discussion on non-key-based notions.

We would like to point out that KCR functions $f_{k}$ can easily be obtained from collision resistant functions $g_{k}$ by defining $f_{k}(x)=g_{x}(k)$. If we require $f$ to inherit the PRF property of $g$, we have to assume that the compression function of $g$ is dual-PRF, meaning that it is a PRF regardless of which input it is keyed with. This is also a requirement of the security proof of HMAC [1]. SKR functions can be constructed equivalently while the KOW property is immediately implied by the PRF property. While we have shown the separation of PRF and KCR, we leave the suspected separation of PRF and SKR as an open problem. Moreover, we have studied the relation between the security level of a PRF and the maximum number of key collisions that can occur.

\section{References}

1. Mihir Bellare. New proofs for nmac and hmac: Security without collision-resistance. In Advances in Cryptology - CRYPTO 2006, volume 4117 of Lecture Notes in Computer Science, pages 602-619. Springer, 2006.

2. Mihir Bellare, Joe Kilian, and Phillip Rogaway. The security of the cipher block chaining message authentication code. Journal of Computer and System Sciences, 61(3):362-399, 2000.

3. Daniel Bleichenbacher and Ueli M. Maurer. Directed acyclic graphs, one-way functions and digital signatures. In Advances in Cryptology - CRYPTO '94, volume 839 of Lecture Notes in Computer Science, pages 75-82, 1994.

4. Johannes Buchmann, Erik Dahmen, Elena Klintsevich, Katsuyuki Okeya, and Camille Vuillaume. Merkle signatures with virtually unlimited signature capacity. In Jonathan Katz and Moti Yung, editors, ACNS, volume 4521 of $L N C S$, pages 31-45. Springer, 2007.

5. Ran Canetti, Daniele Micciancio, and Omer Reingold. Perfectly one-way probabilistic hash functions (preliminary version). In STOC, pages 131-140, 1998.

6. L. C. Coronado García. On the security and the efficiency of the merkle signature scheme. Technical Report 2005/192, Cryptology ePrint Archive, 2005. Available at http://eprint.iacr.org/2005/192/.

7. Erik Dahmen, Katsuyuki Okeya, Tsuyoshi Takagi, and Camille Vuillaume. Digital signatures out of secondpreimage resistant hash functions. In 2nd International Workshop on Post-Quantum Cryptography (PQCrypto), volume 5299 of Lecture Notes in Computer Science, pages 109-123, 2008.

8. Chris Dods, Nigel Smart, and Martijn Stam. Hash based digital signature schemes. In Cryptography and Coding, pages 96-115. Springer Verlag LNCS 3796, November 2005.

9. Marc Fischlin. Pseudorandom function tribe ensembles based on one-way permutations: Improvements and applications. In EUROCRYPT, pages 432-445, 1999.

10. Oded Goldreich, Shafi Goldwasser, and Silvio Micali. How to construct random functions. J. ACM, 33(4):792-807, 1986.

11. Shafi Goldwasser, Silvio Micali, and Ronald L. Rivest. A digital signature scheme secure against adaptive chosenmessage attacks. SIAM J. Comput., 17(2):281-308, 1988. 
12. Lov K. Grover. A fast quantum mechanical algorithm for database search. In Proceedings of the Twenty-Eighth Annual Symposium on the Theory of Computing, pages 212-219, New York, 1996. ACM Press.

13. Alejandro Hevia and Daniele Micciancio. The provable security of graph-based one-time signatures and extensions to algebraic signature schemes. In Advances in Cryptology - ASIACRYPT 2002, volume 2501 of Lecture Notes in Computer Science, pages 379-396, 2002.

14. Leslie Lamport. Constructing digital signatures from a one way function. Technical Report SRI-CSL-98, SRI International Computer Science Laboratory, 1979.

15. Leonid Levin. One way functions and pseudorandom generators. Combinatorica, 7:357-363, 1987. $10.1007 / \mathrm{BF} 02579323$.

16. Mark Luk, Adrian Perrig, and Bram Whillock. Seven cardinal properties of sensor network broadcast authentication. In ACM Workshop on Security of Ad Hoc and Sensor Networks (SASN), 2006.

17. Ralph C. Merkle. A certified digital signature. In Gilles Brassard, editor, CRYPTO, volume 435 of $L N C S$, pages 218-238. Springer, 1989.

18. Adrian Perrig. The biba one-time signature and broadcast authentication protocol. In ACM Conference on Computer and Communications Security, pages 28-37, 2001.

19. Adrian Perrig, Ran Canetti, J. D. Tygar, and Dawn Xiaodong Song. Efficient authentication and signing of multicast streams over lossy channels. In IEEE Symposium on Security and Privacy, pages 56-73, 2000.

20. Leonid Reyzin and Natan Reyzin. Better than biba: Short one-time signatures with fast signing and verifying. In Lynn Batten and Jennifer Seberry, editors, Information Security and Privacy, volume 2384 of Lecture Notes in Computer Science, pages 1-47. Springer Berlin / Heidelberg, 2002.

21. Phillip Rogaway and Thomas Shrimpton. Cryptographic hash-function basics: Definitions, implications, and separations for preimage resistance, second-preimage resistance, and collision resistance. In FSE, pages 371-388, 2004.

22. John Rompel. One-way functions are necessary and sufficient for secure signatures. In STOC '90: Proceedings of the twenty-second annual ACM symposium on Theory of computing, pages 387-394, New York, NY, USA, 1990. ACM Press.

23. Peter W. Shor. Algorithms for quantum computation: Discrete logarithms and factoring. In Proceedings of the 35th Annual IEEE Symposium on Foundations of Computer Science (FOCS 1994), pages 124-134. IEEE Computer Society Press, 1994.

24. Andrew C. Yao. Theory and application of trapdoor functions. Foundations of Computer Science, Annual IEEE Symposium on, 0:80-91, 1982.

\section{A Proof of Theorem 2}

To prove part a) we show in Algorithm 2 how a forger For ${ }^{\text {Sign(sk, }} \cdot(\mathrm{pk})$ for W-OTS can be used to construct an adversary $\mathrm{A}_{\mathrm{SKR}}$ on the second key resistance of $F(n)$ with non negligible advantage. The signing oracle Sign is simulated by the adversary.

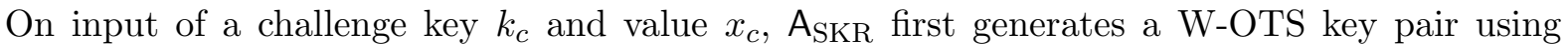
$x=x_{c}$. Then he places $k_{c}$ randomly in the hash chain used to compute $\mathrm{pk}_{\alpha}$ at position $\beta$ and runs For ${ }^{\operatorname{Sign}(\mathrm{sk}, \cdot)}(\mathrm{pk})$ on input $\mathrm{pk}=\left(x_{c}, \mathrm{pk}_{1}, \ldots, \mathrm{pk}_{\ell}\right)$. If the forger queries the oracle on message $M, \mathrm{~A}_{\mathrm{SKR}}$ can only answer the query if $b_{\alpha} \geq \beta$. In this case the adversary returns $(M, \sigma)$ otherwise he returns fail. If the forger succeeds in computing a valid signature $\left(M^{\prime}, \sigma^{\prime}\right)$ there are two possible cases.

If For ${ }_{W-O T S}$ returns a signature for the message sent to the oracle, there has to be at least one index $i$ such that $\sigma_{i} \neq \sigma_{i}^{\prime}$ because $\sigma \neq \sigma^{\prime}$. A SKR only found a 2nd key if (1) $\alpha$ is one of these indices, (2) $b_{\alpha}=\beta$ and (3) $f_{k_{c}}\left(x_{c}\right)=f_{\sigma_{\alpha}^{\prime}}\left(x_{c}\right)$. Observe that (1) implies that $k_{c} \neq \sigma_{\alpha}^{\prime}$ and therefore $\sigma_{\alpha}^{\prime}$ is a 2nd key for the challenge $\left(k_{c}, x_{c}\right)$. So A SkR returns $\sigma_{\alpha}^{\prime}$.

If the forger returns a signature for a new message the adversary can only find a 2nd key for the challenge $k_{c}$ if (1a) $b_{\alpha}^{\prime}<\beta$ or (1b) $b_{\alpha}^{\prime}=\beta$ and $b_{\alpha}>\beta$, if (2) $f_{k_{c}}\left(x_{c}\right)=f_{\sigma_{\alpha}^{\prime}}^{\beta-b_{\alpha}^{\prime}+1}\left(x_{c}\right)$ holds and

last but not least if $k_{c} \neq f_{\sigma_{\alpha}^{\prime}}^{\beta-b_{\alpha}^{\prime}}\left(x_{c}\right)$. If all of these conditions are fulfilled A $\mathrm{A}_{\mathrm{SKR}}$ returns $f_{\sigma_{\alpha}^{\prime}}^{\beta-b_{\alpha}^{\prime}}\left(x_{c}\right)$ as 2nd key. Otherwise $A_{\mathrm{SKR}}$ returns fail. 


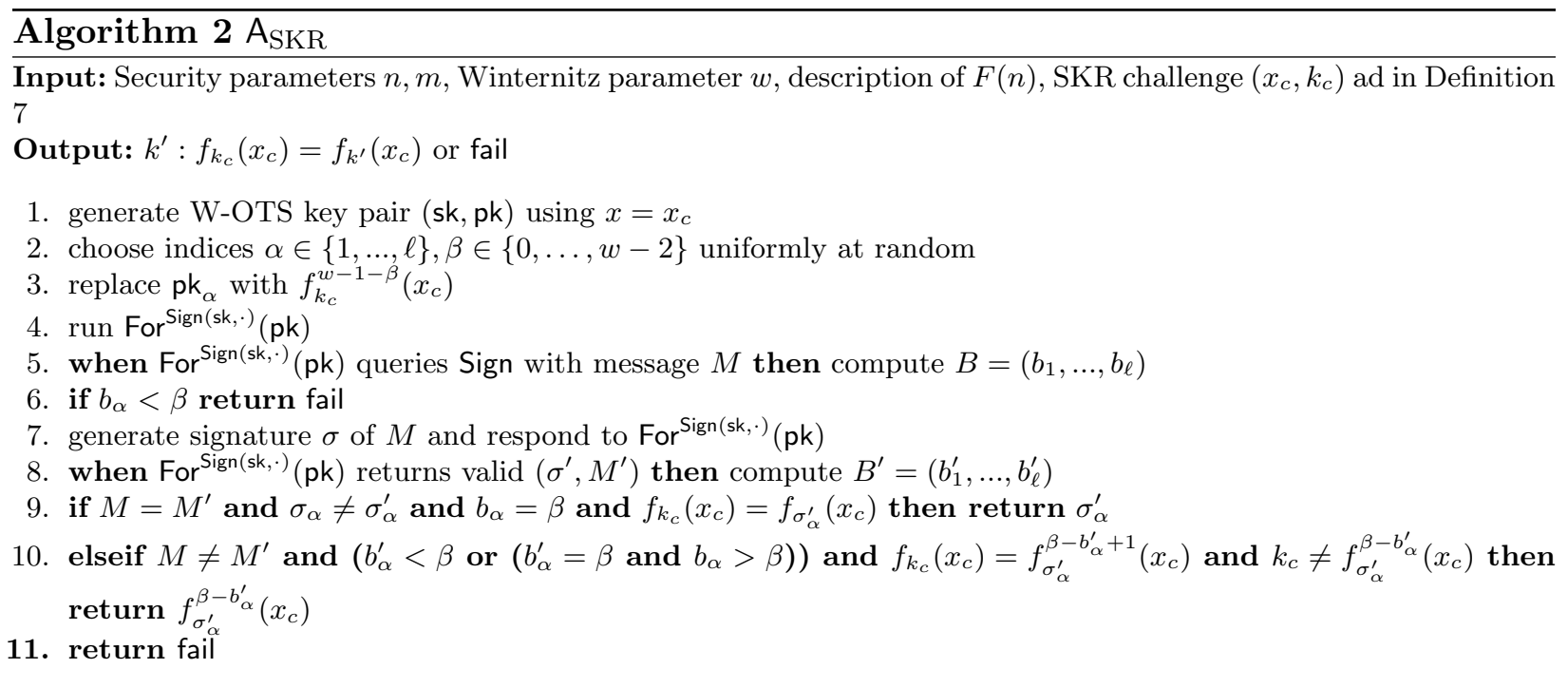

We now compute the success probability. Like in the proof of Theorem 1 a), pk is a possible public key only with probability at least $1 / \kappa^{\beta}$. As before we assume that For ${ }^{\operatorname{Sign}(\mathrm{sk}, \cdot)}(\mathrm{pk})$ queries the oracle without loss of generality. So the probability of $b_{\alpha} \geq \beta$ in line 6 is at least $(\ell w)^{-1}$. This is caused by the checksum which guarantees that at least one $b_{i}$ is greater zero. The forger succeeds with probability at least $\epsilon$ according to the definition. Then we've got two mutual exclusive cases.

Case $1\left(M=M^{\prime}\right)$ : The probability that $\sigma_{\alpha} \neq \sigma_{\alpha}^{\prime}$ holds in line 9 is at least $1 / \ell$ as there has to be at least one index $i$ such that $\sigma_{i} \neq \sigma_{i}^{\prime}$ because $\sigma \neq \sigma^{\prime}$. For the second condition $b_{\alpha}=\beta$ we get a probability of $1 / w$ as $\beta$ was chosen at random. If the second condition holds, the last condition $f_{k_{c}}\left(x_{c}\right)=f_{\sigma_{\alpha}^{\prime}}\left(x_{c}\right)$ holds at least with probability $\frac{1}{\kappa^{w-1-\beta-1}}$, because there are $\left|\left\{y \in\{0,1\}^{n} \mid f_{y}^{w-1-\beta-1}\left(x_{c}\right)=\mathrm{pk}_{i}\right\}\right|=\kappa^{w-1-\beta-1}$ possible values for $f_{\sigma_{\alpha}^{\prime}}\left(x_{c}\right)$. So altogether we get a probability of at least $\frac{\epsilon}{\ell^{2} w^{2} \kappa^{w-2}}$ for finding a 2 nd key for this case.

Case $2\left(M \neq M^{\prime}\right)$ : The probability in Line 10 that $\left(b_{\alpha}^{\prime}<\beta\right.$ or $\left(b_{\alpha}^{\prime}=\beta\right.$ and $\left.\left.b_{\alpha}>\beta\right)\right)$ holds is greater than $(\ell w)^{-1}$. This is because of $M \neq M^{\prime}$ and the checksum which guarantees that $b_{i}>b_{i}^{\prime}$ for some $i \in\{1, \ldots, \ell\}$. Next $f_{k_{c}}\left(x_{c}\right)=f_{\sigma_{\alpha}^{\prime}}^{\beta-b_{\alpha}^{\prime}+1}\left(x_{c}\right)$ holds at least with probability $\frac{1}{\kappa^{w-1-\beta-1}}$ as before. And at last $k_{c} \neq f_{\sigma_{\alpha}^{\prime}}^{\beta-b_{\alpha}^{\prime}}\left(x_{c}\right)$ holds with probability at least $\frac{\kappa^{\prime}-1}{\kappa^{\prime}}$ if the previous condition already holds. Therefore we get a success probability of $\frac{\epsilon\left(\kappa^{\prime}-1\right)}{\ell^{2} w^{2} \kappa^{w-2} \kappa^{\prime}}$ for case 2 .

Since both cases are mutually exclusive, the success probability of $A_{\text {SKR }}$ is

$$
\epsilon_{\mathrm{SKR}} \geq \min \left\{\frac{\epsilon}{\ell^{2} w^{2} \kappa^{w-2}}, \frac{\epsilon\left(\kappa^{\prime}-1\right)}{\ell^{2} w^{2} \kappa^{w-2} \kappa^{\prime}}\right\}=\frac{\epsilon\left(\kappa^{\prime}-1\right)}{\ell^{2} w^{2} \kappa^{w-2} \kappa^{\prime}} .
$$

The time required by $A_{\mathrm{SKR}}$ is $t_{\mathrm{SKR}} \leq t+t_{\mathrm{Kg}}+t_{\mathrm{Vf}}$. The time to answer the signature query is already contained in the runtime of the forger. This concludes the proof of part a).

To prove part b) we show in Algorithm 3 how a forger For ${ }^{\operatorname{Sign}(\mathbf{s k}, \cdot)}$ (pk) for W-OTS can be used to construct an adversary $A_{K C R}$ on the key collision resistance with non negligible advantage. Again, the signing oracle Sign is simulated by the adversary.

The goal of adversary $\mathrm{A}_{\mathrm{KCR}}$ on input $x_{c}$ is to find two different keys $k_{1}, k_{2}$ for which $f_{k_{1}}\left(x_{c}\right)=$ $f_{k_{2}}\left(x_{c}\right)$ holds. Therefore $\mathrm{A}_{\mathrm{KCR}}$ begins by generating a W-OTS key pair using $\left(x=x_{c}\right)$ and calling 


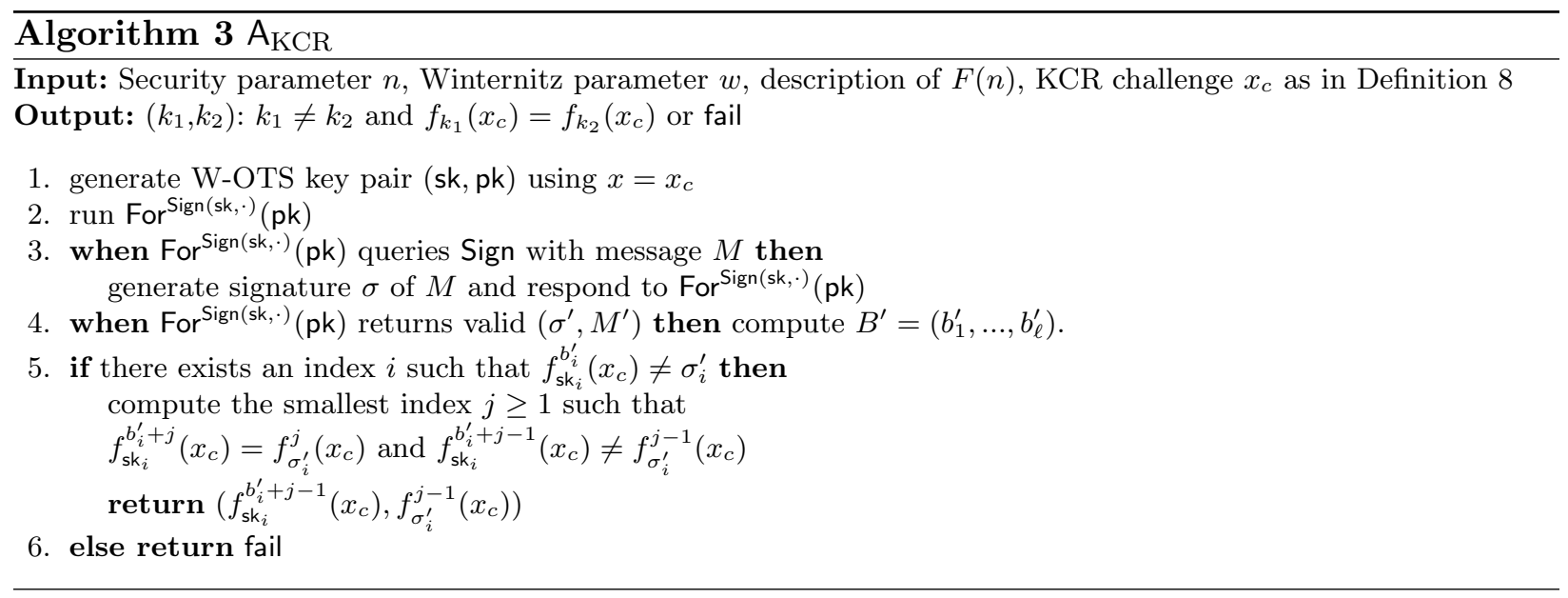

For $^{\operatorname{Sign}(\mathrm{sk}, \cdot)}(\mathrm{pk})$ with the generated public key. If the forger queries the signing oracle Sign for the signature of a message $M, \mathrm{~A}_{\mathrm{KCR}}$ answers this query with $\sigma=\operatorname{Sign}(\mathrm{sk}, M)$. If the forger succeeds in generating a signature $\left(M^{\prime}, \sigma^{\prime}\right), \mathrm{A}_{\mathrm{KCR}}$ computes the vector $B^{\prime}=\left(b_{i}^{\prime}\right), 1 \leq i \leq l$ using $M^{\prime}$ as in the signing algorithm of W-OTS. Then he checks if there exists an index $i$ with $f_{\mathrm{sk}_{i}}^{b_{i}^{\prime}}\left(x_{c}\right) \neq \sigma_{i}^{\prime}$. As $f_{f_{\mathbf{s k}_{i}}^{b_{i}^{\prime}}}^{w-1-b_{i}^{\prime}}\left(x_{c}\right)=\mathrm{pk}_{i}=f_{\sigma_{i}^{\prime}}^{w-1-b_{i}^{\prime}}\left(x_{c}\right)$ there must exist one $j, 0 \leq j \leq w-1-b_{i}^{\prime}$ with $f_{\mathbf{s k}_{i}}^{b_{i}^{\prime}+j}\left(x_{c}\right)=f_{\sigma_{i}^{\prime}}^{j}\left(x_{c}\right)$ and $f_{\mathrm{sk}_{i}}^{b_{i}^{\prime}+j-1}\left(x_{c}\right) \neq f_{\sigma_{i}^{\prime}}^{j-1}\left(x_{c}\right)$. So $\mathrm{A}_{\mathrm{KCR}}$ returns the key collision $\left(f_{\mathrm{sk}_{i}}^{b_{i}^{\prime}+j-1}\left(x_{c}\right), f_{\sigma_{i}^{\prime}}^{j-1}\left(x_{c}\right)\right)$.

We now compute the success probability. The forger returns a valid signature with probability $\epsilon$ per definition. Then we have two alternative cases:

If $M^{\prime}=M$ the forger returned a different signature for the message $M$ signed by Sign. In this case the forger returned a key collision with probability 1 . As $\sigma \neq \sigma^{\prime}$ there has to be at least one index $i$ with $\sigma_{i} \neq \sigma_{i}^{\prime}$ what implies $f_{\text {sk }_{i}}^{b_{i}^{\prime}}\left(x_{c}\right) \neq \sigma_{i}^{\prime}$.

If $M^{\prime} \neq M$ the probability that we find an index $i$ with $f_{\mathbf{s k}_{i}}^{b_{i}^{\prime}}\left(x_{c}\right) \neq \sigma_{i}^{\prime}$ and therefore a key collision is at least $\left(\kappa^{\prime}-1\right) / \kappa^{\prime}$. If the forger did not query Sign there is at least one index i with $b_{i}^{\prime}>0$ because of the checksum construction. If the forger did query Sign there is at least one index i with $b_{i}^{\prime}<b_{i}$ because of the checksum construction. In both cases there is at least one value $\sigma_{i}^{\prime}$ the adversary was unable to take from prior information. As there are $\kappa^{\prime}$ keys mapping $x_{c}$ to some fix value, there are $\kappa^{\prime j}$ possibilities to map $\sigma_{i}^{\prime}$ to $\mathrm{pk}_{i}=f_{\sigma_{i}^{\prime}}^{j}\left(x_{c}\right)$ for $j=w-1-b_{i}^{\prime}$. So the probability that $f_{\mathbf{s k}_{i}}^{b_{i}^{\prime}}\left(x_{c}\right)=\sigma_{i}^{\prime}$ holds is $\left(\kappa^{\prime j}-1\right) / \kappa^{\prime j}$. So in the worst case we find a collision with probability at least $\left(\kappa^{\prime}-1\right) / \kappa^{\prime}$ as we stated above.

Since both cases are mutually exclusive, the probability $\epsilon_{\mathrm{KCR}}$ of finding a key collision is at least $\epsilon_{\mathrm{KCR}} \geq\left(\left(\kappa^{\prime}-1\right) / \kappa^{\prime}\right) \epsilon$. the time required by $\mathrm{A}_{\mathrm{KCR}}$ is $t_{\mathrm{KCR}} \leq t+t_{\mathrm{Kg}}+t_{\mathrm{Vf}}$. The time to answer the signature query is already contained in the runtime of the forger. This concludes the proof of part b).

\section{B Proofs of implications and separations}

Proof of Proposition 3 (KOW $\nRightarrow \mathrm{PRF}$ ). We construct the function family $F(n)$ as follows: $f_{k}(x):=$ $g(k), \forall k, x \in\{0,1\}^{n} . F(n)$ is not pseudorandom as there exists an distinguisher Dis querying Box 
$t$ times with different values and if Box answers all queries with the same value Dis returns 1 . The success probability of Dis is $\epsilon_{\mathrm{PRF}}=1-\frac{2^{n\left(2^{n}-t\right)}}{2^{n 2^{n}}}=1-\frac{1}{2^{t n}}$ running in time $t . F(n)$ is KOW as we could construct an adversary $\mathrm{A}_{\mathrm{OW}}$ on the one-wayness of $g$ using any adversary $\mathrm{A}_{\mathrm{KOW}}$ on the KOW property of $F(n)$. On input $y$ A ow chooses $x \stackrel{\$}{\longleftarrow}\{0,1\}^{n}$, runs $k \leftarrow \mathrm{A}_{\mathrm{KOW}}(x, y)$ and returns $k$.

Proof of Proposition 4 (KOW $\nRightarrow$ SKR). We denote functions in $F(n)$ and $F^{\prime}(n)$ with $f$ and $f^{\prime}$ respectively, and we define $F^{\prime}(n)$ as follows. For all $k \in\{0,1\}^{n-1}$, we define $f_{k \| 0}^{\prime}:=f_{k \| 0}=: f_{k \| 1}^{\prime}$ and add $\left\{f_{k \| 0}^{\prime}, f_{k \| 1}^{\prime}\right\}$ to $F^{\prime}(n)$. Thus, we have that $f_{k}^{\prime}=f_{k \oplus\left(0^{n-1} \| 1\right)}^{\prime}$ for every $k \in\{0,1\}^{n}$. Observe that $F^{\prime}(n)$ is KOW because a successful adversary against KOW in $F^{\prime}(n)$ will output the correct key w.r.t. $F(n)$ with probability at least $1 / 2$. Given the fact that key-collisions are easy to find, the new family is not SKR. On input $(x, k)$, the adversary simply outputs $k \oplus\left(0^{n-1} \| 1\right)$ and wins with probability 1 in the SKR experiment.

Proof of Proposition 5 (KOW $\nRightarrow$ KCR). Let $k_{1}, k_{2} \in\{0,1\}^{n}$ be distinct, fixed keys and denote functions in $F(n)$ and $F^{\prime}(n)$ with $f$ and $f^{\prime}$ respectively. We define the new family $F^{\prime}(n)$ as follows. For all $k \in\{0,1\}^{n} \backslash\left\{k_{2}\right\}$, we set $f_{k_{1}}^{\prime}:=f_{k_{1}}$ and inject a collision $f_{k_{2}}^{\prime}:=f_{k_{1}}^{\prime}$. The new family is still KOW because the challenge key is chosen uniformly at random and the above change does not influence the adversaries success probability but for a negligible $\left(2 / 2^{n}\right)$ amount. However, the new family is not KCR because on input a description of $F^{\prime}(n)$, the adversary will simply output $\left(k_{1}, k_{2}\right)$, under which every input collides.

Proof of Proposition 6 (KCR $\Rightarrow$ SKR). Towards contradiction, let us assume a successful adversary A that breaks SKR for $F(n)$. We show how to use $\mathrm{A}$ as a block-box in an algorithm $\mathcal{B}$ to break KCR. On input $x$ from the KCR experiment, the algorithm $\mathcal{B}$ chooses $k$ uniformly at random and runs $\mathrm{A}(x, k)$. The subroutine returns $k^{\prime}$ such that $k^{\prime} \neq k$ and $f_{k}(x)=f_{k^{\prime}}(x)$ with probability at least $\epsilon$. Then, $\mathcal{B}$ returns the pair $\left(k, k^{\prime}\right)$ and is successful with the same probability $\epsilon$ and a negligible computational overhead.

Proof of Proposition 7 (SKR $\nRightarrow \mathrm{KCR}$ ). Let $k_{1}, k_{2} \in\{0,1\}^{n}$ be distinct, fixed keys and denote functions in $F(n)$ and $F^{\prime}(n)$ with $f$ and $f^{\prime}$ respectively. We define the new family $F^{\prime}(n)$ as follows. For all $k \in\{0,1\}^{n} \backslash\left\{k_{2}\right\}$, we set $f_{k_{1}}^{\prime}:=f_{k_{1}}$ and inject a collision $f_{k_{2}}^{\prime}:=f_{k_{1}}^{\prime}$. The new family is still SKR because the challenge key is chosen uniformly at random and the above change does not influence the adversaries success probability but for a negligible $\left(2 / 2^{n}\right)$ amount. However, the new family is not KCR because on input a description of $F^{\prime}(n)$, the adversary will simply output $\left(k_{1}, k_{2}\right)$, under which every input collides.

Proof of Proposition 8 ( $\mathrm{PRF} \nRightarrow \mathrm{KCR}$ ). We construct $F^{\prime}$ as follows. We select $k_{1}, k_{2} \stackrel{\$}{\longleftarrow}\{0,1\}^{n}$, and define $f_{k}^{\prime} \in F^{\prime}(n)$ as $x \mapsto\left\{\begin{array}{l}0^{n} \text { for } k \in\left\{k_{1}, k_{2}\right\} \\ f_{k}(x) \text { otherwise }\end{array} \quad\right.$ for all $k \in\{0,1\}^{n}$.

Towards contradiction, let us assume that $F^{\prime}$ is not PRF. Then, there is a distinguisher A that breaks PRF with non-negligible probability $\epsilon$. With access to Box, we construct an adversary Dis against the family $F$. The distinguisher Dis answers all queries of $A$ with its own oracle and simply forwards the output of $\mathrm{A}$ as its decision. Hence, the advantage of Dis is $\epsilon-2 / 2^{n}$ because the probability that Box represents $f_{k_{1}}$ or $f_{k_{2}}$ is at most $2 / 2^{n}$, which contradicts the assumption.

Furthermore, $F^{\prime}$ is clearly not KCR because on input a preimage $x$, one can simply output $\left(k_{1}, k_{2}\right)$ as the "colliding" keys. 